\title{
CARACTERIZACIÓN DE ÁREAS PRODUCTORAS DE SEMILLAS DE ESPECIES PRIORITARIAS PARA LA DIVERSIFICACIÓN FORESTAL ESTABLECIDAS POR EL INSTITUTO FORESTAL
}

\author{
Maria Paz Molina B., Braulio Gutiérrez C., Oriana Ortiz N., Andrés Bello D., Mauricio \\ Navarrete y Juan Carlos Pinilla'
}

\section{RESUMEN}

El trabajo entrega la evaluación y caracterización de Áreas Productoras de Semillas (APS) establecidas por el Instituto Forestal durante el desarrollo de diferentes proyectos de investigación, entre las Regiones de Bio Bio y Aysén, para siete de las once especies consideradas prioritarias para la diversificación forestal en el país.

Se presenta una descripción y caracterización de la situación original y la situación actual de los rodales productores de semillas y una estimación de su capacidad de producción anual en cada caso. El objeto es dar a conocer estas APS, que proporcionan semilla mejorada, como base para promocionar el uso de estas especies y contribuir asi a la diversificación forestal en el pais.

Las APS establecidas son 18 y corresponden a cinco especies nativas; Nothofagus obliqua, roble; Nothofagus dombeyi, coihue; Nothofagus alpina, rauli; Nothofagus pumilio, lenga y Drimys winteri, canelo; y dos especie exóticas; Acacia melanoxylon, aromo australiano; y Pseudotsuga menziesii.

Las restantes especies consideradas prioritarias; Eucalyptus regnans; Pinus pinea, pino piñonero; Castaena sativa, castaño; y Populus spp, álamos. Se está avanzando en el establecimiento de APS para estas especies, con la excepción de álamo que no se la reproduce comercialmente por semillas, las cuales serán establecidas mediante huertos semilleros clonales a partir de ejemplares superiores. 


\section{SUMMARY}

Descriptions of seed production areas established by the Forestry Institute through different research projects, between the Bio Bio and Aysen Regions, for some of the main priority species considered for forest diversification in Chile, are included in this paper.

The characterization of stands before and after the establishment of the seed production area and the estimated seed production are given. Main objective is to inform about this areas as a basis to promote the use of the species and the forest diversification in the country.

Established areas are 18 and include five native species; Nothofagus obliqua, Roble; Nothofagus dombeyi, Coihue; Nothofagus alpina, Rauli; Nothofagus pumilio, Lenga and Drimys winteri, Canelo; and two exotic species; Acacia melanoxylon, Black Wattle; and Pseudotsuga menziesii, Douglas Fir.

The other priority species are Eucalyptus regnans, Mountain Ash; Pinus pinea, Pino Piñonero; Castaena sativa, Chestnut; and Populus spp, Poplar. The Institute is already working on the establishment of seed production areas for these species as well, except for Poplar which is not commercially reproduced by seeds, in this case by the way of clonal seed orchards using plus trees material. 


\section{INTRODUCCIÓN}

El establecimiento de Áreas Productoras de Semillas (APS) es uno de los métodos más apropiados para producir semilla con algún grado de ganancia genética en los inicios de un programa de mejora. Normalmente se afirma que los procedimientos de producción de semilla mejorada previos a los huertos, no permiten obtener beneficios importantes. Efectivamente las ganancias asociadas al uso de Áreas Productoras de Semillas son menores que las derivadas del uso de semilla de huerto, pero aún asi, el sólo hecho de cosechar semilla de fenotipos seleccionados permite obtener ganancias en adaptabilidad y otras caracteristicas, que justifican la implementación de este método, razón por la que la implementación de APS se ha considerado una etapa básica durante el desarrollo de variados programas de mejoramiento.

En la actualidad existen diversas APS que han sido establecidas en proyectos cuya ejecución ya ha terminado. El presente documento presenta un diagnóstico del estado actual de esas APS, y se plantea las intervenciones requeridas para constituirlas en unidades de producción apropiadas para incorporarlas a la red de fuentes semilleras propuestas en el proyecto Fondo SAG (Servicio Agricola y Ganadero) "Fuentes de Semilla Mejorada para las Especies Prioritarias en la Estrategia de Diversificación Forestal Nacional", el cual tiene como objetivo general proveer de semilla genéticamente mejorada de especies forestales alternativas para satisfacer la necesidad estratégica de diversificar la producción forestal del pais.

\section{OBJETIVOS}

El objetivo del establecimiento de fuentes de semilla genéticamente mejorada (APS y huertos semilleros) de especies prioritarias para la diversificación forestal es contribuir a la difusión de estas especies, apoyar programas de plantación con ellas y generar una masa forestal productiva que permita la generación de alternativas de productos al pais.

El objetivo especifico de este documento es caracterizar el estado actual de APS establecidas anteriormente, en el marco de distintos programas de mejoramiento en que ha participado el Instituto Forestal (INFOR), darlas a conocer al sector y promocionar el uso de especies forestales alternativas en la producción forestal nacional mediante material genético con algún grado de mejoramiento.

\section{MATERIAL Y MÉTODO}

El diagnóstico de las APS existentes se realizó mediante el análisis de 14 unidades que fueron establecidas durante la ejecución de programas de mejoramiento genético realizados por INFOR y otras instituciones (Universidad Austral, Corporación Nacional Forestal y Empresas Forestales) y 4 unidades establecidas durante la ejecución del proyecto SAG. La administración actual corresponde a INFOR, quien cuenta con financiamiento del proyecto señalado para mantener y aprovechar estas unidades. Las APS establecidas corresponden a las identificadas en el Cuadro $\mathrm{N}^{\circ} 1$. 
El diagnóstico consistió en:

Evaluar la metodología empleada para establecer las APS

Caracterizar su estado actual

Comparar su estado actual con el estado inicial del rodal y con las caracteristicas de la APS proyectada.

\section{Cuadro $\mathrm{N}^{\circ} 1$}

ÁREAS PRODUCTORAS DE SEMILLAS (APS) CONSIDERADAS EN EL PROYECTO FONDO SAG

\begin{tabular}{|c|c|c|}
\hline ESPECIE & $N^{\circ}$ DE UNIDADES & PREDIO (Propietario) \\
\hline Coigüe & 1 & Pilmaiquèn (COFOMAP) \\
\hline \multirow{3}{*}{ Pino oregón } & \multirow{3}{*}{3} & Aquilhue (Agricola y Forestal Taquihue Ltda.) \\
\hline & & Pilmaiquén (COFOMAP) \\
\hline & & Malacahuello (CONAF) \\
\hline \multirow{4}{*}{ Rauli } & \multirow{4}{*}{4} & El Morro (JCE Ltda.) \\
\hline & & Malalcahuello (CONAF) \\
\hline & & El Manzano (MAGASA) \\
\hline & & Remeco (Forestal Neltume Carranco S.A.) \\
\hline \multirow{5}{*}{ Roble } & \multirow{5}{*}{5} & Aquilhue (Agricola y Forestal Taquihue Ltda.) \\
\hline & & Fuy (COFOMAP) \\
\hline & & Parcela 7 (Sucesión Zúñiga) \\
\hline & & Pumillahue (CONAF) \\
\hline & & Rupanco ( Manuka. ex Forestal Cabildo S.A.) \\
\hline Lenga & 1 & Caiquén Grande, Forestal Mininco \\
\hline \multirow{2}{*}{ Canelo } & \multirow{2}{*}{2} & Río Sur, José Soto Santana, Puerto Varas \\
\hline & & Aguas Buenas, Helga Montecinos Araya, Ancud \\
\hline \multirow{2}{*}{ Aromo Australiano } & \multirow{2}{*}{2} & Quilas Bajas, Hernan Toepser Klima \\
\hline & & Buchoco, Herito Leal Carrillo \\
\hline
\end{tabular}

\section{Metodologia para Establecer APS}

Se efectuó un análisis de los procedimientos empleados para establecer APS que han sido reportados en distintas investigaciones de mejoramiento genético forestal. En general estos planteamientos corresponden a su aplicación en plantaciones, por lo cual, en el caso del bosque nativo, se hizo necesario adecuarlos al ecosistema natural. El procedimiento empleado se describe en los puntos siguientes.

\section{- Selección de Rodales Candidatos}

Sobre la base de antecedentes generales recabados en investigaciones anteriores, información de empresas y experiencias personales, se determinaron zonas de prospección donde la calidad del bosque existente podria permitir la creación de una APS. Como se verá en los párrafos posteriores, la formación de una APS involucra la ejecución de labores silvicolas que implican la eliminación de árboles, por lo cual, la elección final del rodal considera el total 
acuerdo con el propietario.

\section{- Caracterización de los Rodales Candidatos}

Para efectos de comparar entre si los rodales candidatos y poder seleccionar en forma más objetiva a aquellos que se transformarian en APS, se procedió a caracterizar a cada uno de ellos mediante el planteamiento de parcelas de muestreo.

De acuerdo a lo anterior se construyó parcelas circulares de 1/20 de hectárea (radio $=12,61 \mathrm{~m}$ ), las que se distribuyó al azar, con una intensidad de muestreo del $5 \%$ ( 1 parcela por hectárea de rodal candidato). En ellas se evaluó a todos los árboles, registrándose antecedentes dasométricos y fundamentalmente variables relacionadas con la calidad y forma de ellos (Cuadro $\mathrm{N}^{\circ} 2$ ).

Con la información de estas parcelas se procedió a calcular los parámetros descriptivos del rodal. De este modo se construyó un índice de calidad (IC) de cada árbol, el cual se definió como la suma lineal de los puntajes obtenidos en las variables rectitud de fuste, copa, ángulo de ramas y diámetro de ramas, ponderando por 3 la rectitud de fuste y posteriormente transformado a una escala de 0 a 100.

$$
I C \%=3 x R F+C+D R+A R(0 \text { a } 100)
$$

Cabe hacer notar que la rectitud del fuste se torna en una variable preponderante, debido a que es una característica altamente heredable por la descendencia generada a través de semillas y es también un factor significativo en el aprovechamiento industrial de la madera.

\section{Cuadro $\mathrm{N}^{\circ} 2$ \\ PAUTA DE EVALUACIÓN DE ÁRBOLES PARA CARACTERIZACIÓN DE RODALES CANDIDATOS A APS}

\begin{tabular}{|c|c|}
\hline $\begin{array}{l}\text { PS: Posición Social } \\
\text { 1. Suprimido o Intermedio } \\
\text { 2. Dominante o codominante }\end{array}$ & $\begin{array}{l}\text { DAP: Diámetro Altura del Pecho } \\
\qquad(\mathrm{cm})\end{array}$ \\
\hline $\begin{array}{l}\text { Ht: Altura Total } \\
\text { (m) }\end{array}$ & $\begin{array}{l}\text { Hc: Altura Comercial. } \\
\qquad(\mathrm{m})\end{array}$ \\
\hline & $\begin{array}{l}\text { R.F. Rectitud Fuste } \\
\text { 1. Torcedura severa con pérdida del eje. } \\
\text { 2. Torcedura pronunciada en un plano y otras } \\
\text { 3. Torcedura pronunciada en un plano sin pérdida del eje } \\
\text { 4. Torceduras leves en más de un plano } \\
\text { 5. Recto o con torcedura leve en un sölo plano }\end{array}$ \\
\hline
\end{tabular}




\begin{tabular}{|c|c|}
\hline C: Copa & \multirow{3}{*}{$\begin{array}{l}\text { 1. Muy inferior al promedio } \\
\text { 2. Inferior al promedio } \\
\text { 3. Promedio } \\
\text { 4. Superior al promedio } \\
\text { 5. Muy superior al promedio }\end{array}$} \\
\hline DR: Diámetro de ramas & \\
\hline AR: Ángulo de ramas & \\
\hline BIF: Bifurcaciones & SAN: Sanidad \\
\hline $\begin{array}{l}\text { 1. Bifurcación en fuste comercial } \\
\text { 2. Sin Bifurcación, o sobre altura comercial }\end{array}$ & $\begin{array}{l}\text { 1. Daños generalizados } \\
\text { 2. Signos locales de daño } \\
\text { 3. Aparentemente sano }\end{array}$ \\
\hline
\end{tabular}

\section{- Transformación de los Rodales en APS}

Una vez caracterizados los rodales a transformar en APS, se simuló la conformación de la APS mediante la eliminación de los datos de los individuos menos deseables y se procedió a recalcular los parámetros de rodal y coeficiente de calidad en base a los árboles superiores remanentes. Posteriormente, se marca en terreno un raleo utilizando el mismo criterio que se usó para simular la transformación del rodal, pero con la información generada en las nuevas parcelas de muestreo.

El paso siguiente lo constituye la ejecución del raleo, el manejo de los desechos y la identificación de las zonas de cosecha y aislación (Figura $\mathrm{N}^{\circ} 1$ ). La primera zona corresponde al núcleo de la APS donde efectivamente se puede cosechar semilla mejorada que de algún modo refleje la calidad de los padres (árboles remanentes). La zona de aislación es un área de protección donde se evita el ingreso de polen indeseable de otros árboles aledaños a la APS. A esta zona también se le denomina área de dilución de polen y no se considera cuando se señala la superficie de la APS, aún cuando los árboles presentes en esta área tienen la misma calidad que los árboles de la zona de cosecha.

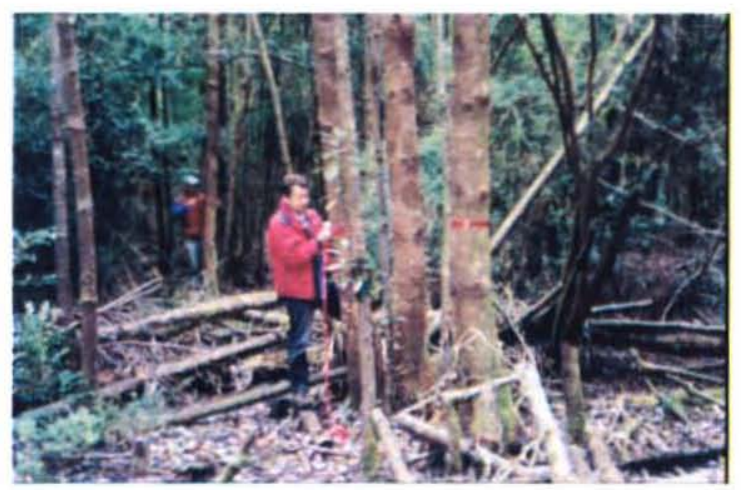

Figura $\mathrm{N}^{\circ} 1$

TRANSFORMACIÓN APS 


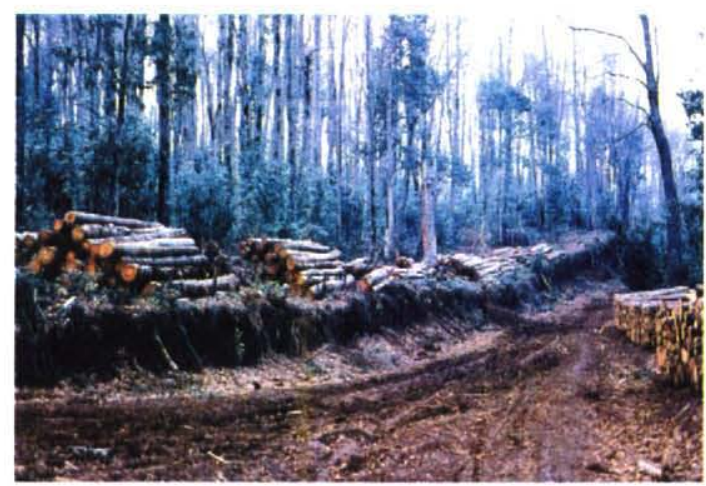

Figura $\mathrm{N}^{\circ} 2$

EVALUACIÓN Y MARCACIÓN DE ÁRBOLES EN APS

\section{Estado Actual de las APS}

Fueron reevaluadas las APS establecidas haciendo uso de técnicas de muestreo equivalentes a las utilizadas originalmente en los rodales (parcelas circulares de $500 \mathrm{~m}^{2}$ distribuidas con una intensidad de muestreo de $5 \%$ equivalente a una parcela por hectárea). Las parcelas fueron distribuidas al azar y se registró datos dasométricos y de calidad de los árboles. El procedimiento de muestreo fue el mismo que para la caracterización inicial de los rodales seleccionados y que se describió en el punto anterior. Los datos registrados se han expresado en términos de parámetros de rodal y de caracteristicas de calidad resumidas en el indice de calidad promedio. Dentro de lo posible, se desplegó las parcelas de este muestreo sobre los mismos lugares en que se estableció el muestreo original. La operación se efectuó en verano del año 2004. Utilizando igual metodologia se evaluó 3 rodales candidatos de aromo australiano y 3 de canelo.

\section{Comparación de Estado Actual con Situación Original y Proyectada}

Se comparó el estado actual de las APS con los datos registrados al momento de seleccionar el rodal. Esto se extendió también a la situación proyectada, mediante simulación de las intervenciones de raleo. Esta comparación permite determinar si el manejo aplicado mejora efectivamente la calidad del rodal y cuanto más falta intervenir para llegar al escenario final proyectado.

\section{DESCRIPCIÓN GENERAL DE LAS ÁREAS PRODUCTORAS DE SEMILLAS}

El procedimiento implementado para el establecimiento de APS fue homogéneo para todas las unidades. Se seleccionó rodales candidatos, se evaluó en función de la calidad de los árboles, se simuló el estado de la APS resultante y posteriormente, en función de características dasométricas y de calidad del rodal remanente, se identificó los rodales que definitivamente se transformarian en APS. Una vez definidos los rodales se procede a su conversión mediante raleos sucesivos (Figura $\mathrm{N}^{\circ} 2$ ). 
Se simuló la aplicación de raleos necesarios para la conversión definitiva de los rodales en APS. La ejecución de estos raleos se distanció 2 a 3 años en cada APS. En la mayoria de los casos se efectuó todas las intervenciones, como consecuencia el estado actual de las APS se transforma en el estado final y, como se puede apreciar en el Cuadro $\mathrm{N}^{\circ} 3$, los índices de calidad promedio de los árboles aumentaron. En algunos casos existia la posibilidad de realizar un mayor número de raleos, lo que permitiría mejorar aún más el valor de este indice de calidad, sin embargo se ponia en riesgo la permanencia de la APS debido al aumento de la vulnerabilidad del rodal al viento. Cabe señalar que el proceso de intervenciones se realizó paulatinamente durante periodo aproximado de 8 años, al inicio del cual se parte con las APS generadas en programas de mejoramiento genético anteriores de INFOR en conjunto con otras instituciones. Durante los últimos 4 años se finalizó las intervenciones indicadas para todas las APS existentes y se llevó a cabo el procedimiento total de generación de un Área Productora de Semillas para las especies aromo australiano y canelo.

Cabe señalar que las APS de rauli de El Morro y Parcela 2 de roble, si bien fueron evaluadas para definir su transformación, finalmente no fueron establecidas. El rodal seleccionado denominado El Morro, luego de la simulación de las intervenciones no presentaba una mejora que justificara la intervención. En el caso de la APS denominada Parcela 7 no hubo acuerdo con el propietario para la intervención.

\section{Cuadro $\mathrm{N}^{\circ} 3$ \\ SITUACIÓN ORIGINAL Y ACTUAL DE CADA APS}

\begin{tabular}{|c|c|c|c|c|c|c|}
\hline Especie/Predio & Estado & $\begin{array}{l}\text { Superficie } \\
\text { (ha) }\end{array}$ & $\begin{array}{c}\text { Densidad } \\
\text { (árb/ha) }\end{array}$ & $\begin{array}{l}\text { DAP } \\
(\mathrm{cm})\end{array}$ & $\begin{array}{l}\mathrm{Hc} \\
(\mathrm{m})\end{array}$ & $\begin{array}{c}\text { IC } \\
(0-100)\end{array}$ \\
\hline \multirow{3}{*}{ Coigue/Pilmaiquén } & Original & & 645 & 27,5 & 14 & 30,1 \\
\hline & Actual & 7 & 233 & 40,1 & 19,5 & 48,2 \\
\hline & Aumento IC & & & & & $60 \%$ \\
\hline \multirow{3}{*}{ Rauli/Malalcahuello } & Original & & 240 & 41,1 & 19,3 & 34.5 \\
\hline & Actual & 3 & 60 & 44 & 20,8 & 48.1 \\
\hline & Aumento IC & & & & & $39 \%$ \\
\hline \multirow{3}{*}{ Rauli/EI Manzano } & Original & & 490 & 28 & 18.9 & 36,8 \\
\hline & Actual & 10 & 300 & 31,7 & 20,5 & 51 \\
\hline & Aumento IC & & & & & $39 \%$ \\
\hline \multirow{3}{*}{ Rauli/Remeco } & Original & & 300 & 31,5 & 16 & 26,4 \\
\hline & Actual & 6 & 213 & 37,1 & 19,3 & 61,6 \\
\hline & Aumento IC & & & & & $133 \%$ \\
\hline
\end{tabular}




\begin{tabular}{|c|c|c|c|c|c|c|}
\hline Especie/Predio & Estado & $\begin{array}{l}\text { Superficie } \\
\text { (ha) }\end{array}$ & $\begin{array}{c}\text { Densidad } \\
\text { (árb/ha) }\end{array}$ & $\begin{array}{l}\text { DAP } \\
\text { (cm) }\end{array}$ & $\begin{array}{l}\mathrm{Hc} \\
(\mathrm{m})\end{array}$ & $\underset{(0-100)}{\text { IC }}$ \\
\hline \multirow{3}{*}{ Roble/Arquilhue } & Original & & 360 & 35,5 & 22,1 & 34,9 \\
\hline & Actual & 3 & 173 & 42,2 & 26,3 & 49,8 \\
\hline & Aumento IC & & & & & $43 \%$ \\
\hline \multirow{3}{*}{ Roble/Fuy } & Original & & 280 & 29,5 & 18,7 & 32,7 \\
\hline & Actual & 2,1 & 210 & 34,2 & 23,1 & 48 \\
\hline & Aumento IC & & & & & $47 \%$ \\
\hline \multirow{3}{*}{ Roble/Pumillahue } & Original & & 640 & 26,9 & 19,1 & 37,8 \\
\hline & Actual & 3,5 & 247 & 35,2 & 23.1 & 47,1 \\
\hline & Aumento IC & & & & & $25 \%$ \\
\hline \multirow{3}{*}{ Roble/Rupanco } & Original & & 460 & 28,9 & 19,6 & 29,5 \\
\hline & Actual & 1,8 & 140 & 40,7 & 25 & 52,4 \\
\hline & Aumento IC & & & & & $78 \%$ \\
\hline \multirow{3}{*}{ Lenga/Caiquen } & Original & & 670 & 35,6 & 10,2 & 43,8 \\
\hline & Actual & 6 & 175 & 43,3 & 11,7 & 53,6 \\
\hline & Aumento IC & & & & & $22 \%$ \\
\hline \multirow{3}{*}{ Canelo/Rio Sur } & Original & & 1386 & 15,9 & 10,1 & 56,2 \\
\hline & Actual & 2,5 & 373 & 18,1 & 11,2 & 77 \\
\hline & Aumento IC & & & & & $37 \%$ \\
\hline \multirow{3}{*}{ Canelo/Aguas Buenas } & Original & & 2180 & 13 & 10,2 & 49,1 \\
\hline & Actual & 2 & 320 & 16,1 & 10,2 & 77 \\
\hline & Aumento IC & & & & & $57 \%$ \\
\hline \multirow{3}{*}{ Pino Oregón/Arquilhue } & Original & & 760 & 28,2 & 15 & 42,3 \\
\hline & Actual & 3 & 310 & 39 & 22,4 & 66,9 \\
\hline & Aumento IC & & & & & $58 \%$ \\
\hline \multirow{3}{*}{ Pino Oregón/Pilmaiquen } & Original & & 600 & $S / 1$ & $\mathrm{~S} / \mathrm{l}$ & $S / I$ \\
\hline & Actual & 7 & 366 & 34 & 15,2 & 58,5 \\
\hline & Aumento IC & & & & & $S / 1$ \\
\hline
\end{tabular}




\begin{tabular}{|c|c|c|c|c|c|c|}
\hline Especie/Predio & Estado & $\begin{array}{l}\text { Superficie } \\
\text { (ha) }\end{array}$ & $\begin{array}{c}\text { Densidad } \\
\text { (ärb/ha) }\end{array}$ & $\begin{array}{l}\text { DAP } \\
(\mathrm{cm})\end{array}$ & $\begin{array}{l}\mathrm{Hc} \\
(\mathrm{m})\end{array}$ & $\underset{(0-100)}{\text { IC }}$ \\
\hline \multirow{3}{*}{$\begin{array}{l}\text { Pino Oregón/ } \\
\text { Malalcahuello }\end{array}$} & Original & & 447 & 35,1 & 16,3 & 41.1 \\
\hline & Actual & 2.7 & 350 & 39,6 & 20,1 & 68 \\
\hline & Aumento IC & & & & & $65 \%$ \\
\hline \multirow{3}{*}{ Acacia/Buchoco } & Original & & 3330 & 12,5 & 12,4 & 34,3 \\
\hline & Actual & 1 & 210 & 21.6 & 14,8 & 68,3 \\
\hline & Aumento IC & & & & & $99 \%$ \\
\hline \multirow{3}{*}{ Acacia/Quilas Bajas } & Original & & 680 & 18.5 & 11,3 & 38,7 \\
\hline & Actual & 2,5 & 110 & 19.1 & 11,8 & 72,3 \\
\hline & Aumento IC & & & & & $87 \%$ \\
\hline
\end{tabular}

(Fuente: Proyecto Fondo SAG: Fuentes de Semilla Mejorada para las Especies Prioritarias en la Estrategia de Diversificación Forestal Nacional).

DAP: Diámetro a la Altura del Pecho promedio.

Hc: Altura comercial Promedio

IC: Índice de calidad promedio (Equivale al promedio de los indices de calidad de cada árbol evaluado).

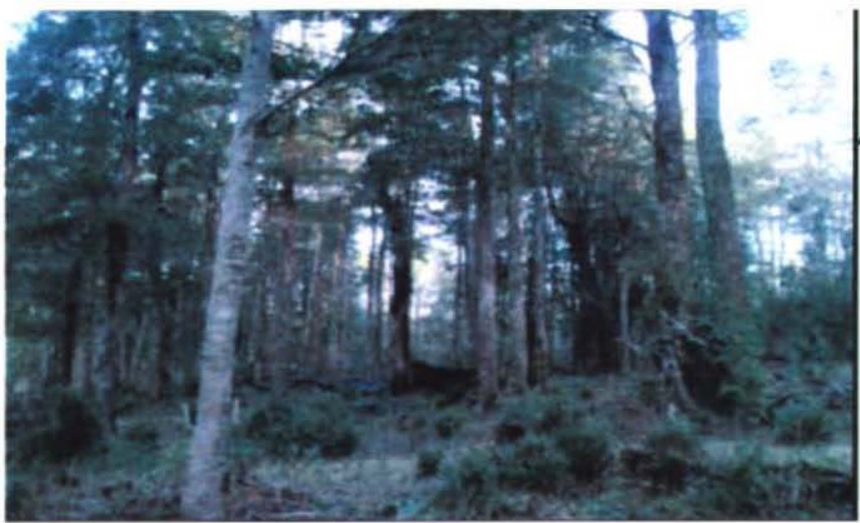

APS COIGÜE PILMAIQUEN

Renoval coigüe

15 ha

Sector "Aserradero" Predio Pilmaiquén

Empresa COFOMAP S.A.

Precordillera Andina

Comuna de Neltume

Región de Los Rios

Los raleos sucesivos conducen a un mejoramiento en el indice de un $60 \%$ 


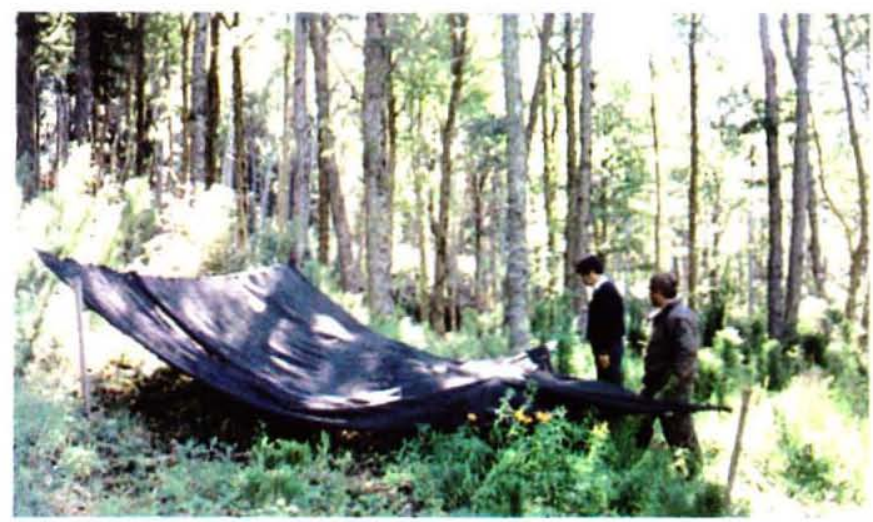

APS RAULI MALALCAHUELLO Rodal rauli 3 ha

Reserva Forestal Malacahuello CONAF

Región de La Araucania

Raleos conducen a mejoramiento en el indice de calidad de un $39 \%$ (Malla para colecta semillas)

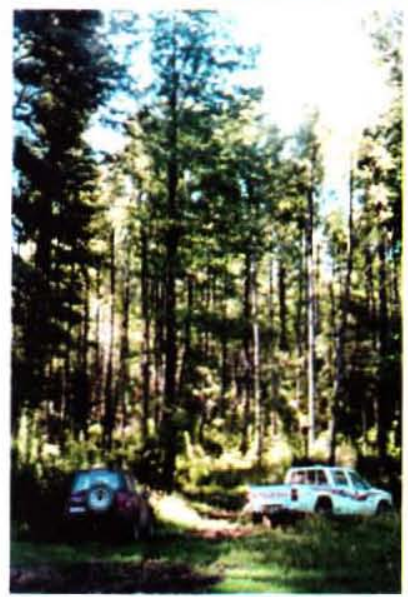

\section{APS RAULI EL MANZANO}

Rodal rauli 40 a 60 años edad

10 ha

Predio El Manzano

Forestal MAGASA SA:

Precordillera Andina

Comuna de Melipeuco

Región de La Araucania

El sector corresponde a una ladera de pendiente moderada,

exposición sur, frente al sector de "Los Mesones"

Índice de calidad aumentó en un 39\%.

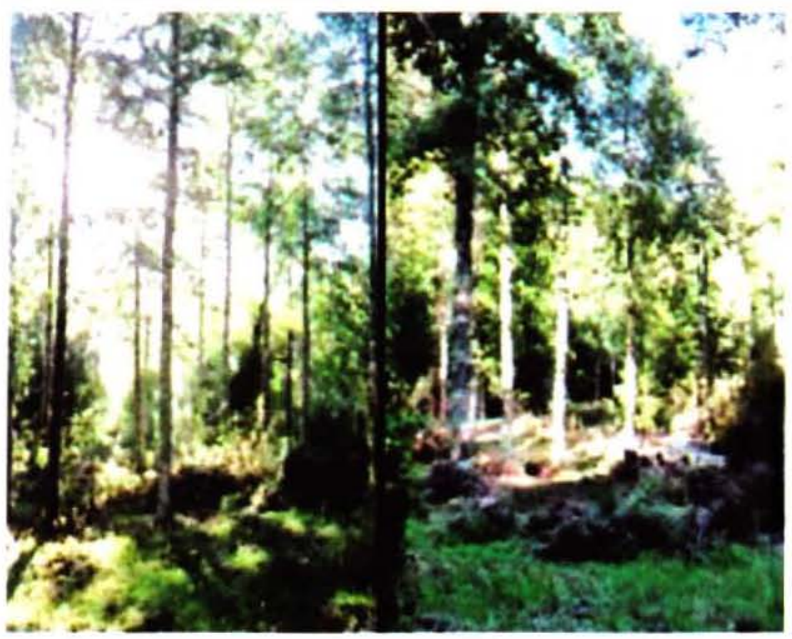

\section{APS RAULI REMECO}

Rodal casi puro rauli 40 a 60 años edad 6 ha

Predio Remeco COFOMAP SA.

Precordillera Andina

Comuna de Neltume

Región de Los Rios 
Índice de calidad aumentó en un $133 \%$

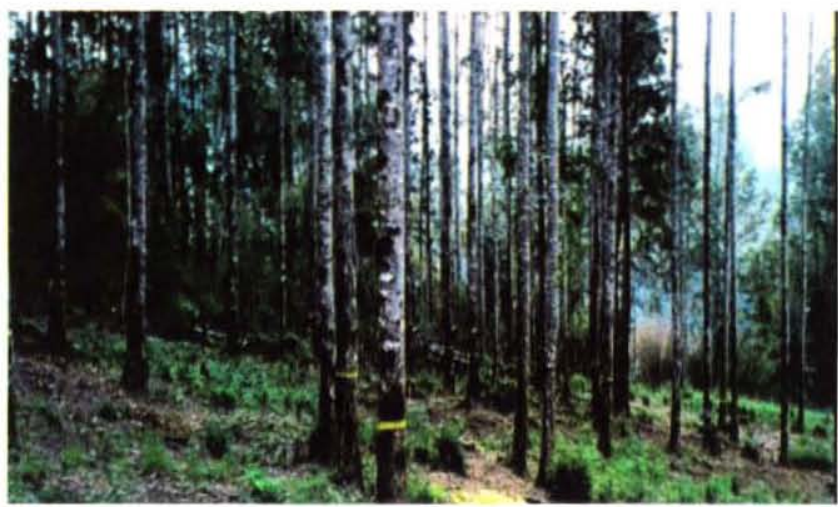

APS ROBLE ARQUILHUE

Renoval roble

4 ha

Predio Arquilhue

Propietario Agricola y Forestal

Taquihue Ltda.

Comuna de Futrono

Región de Los Ríos

Sector "Vivero"

Índice de calidad aumentó en un $39 \%$
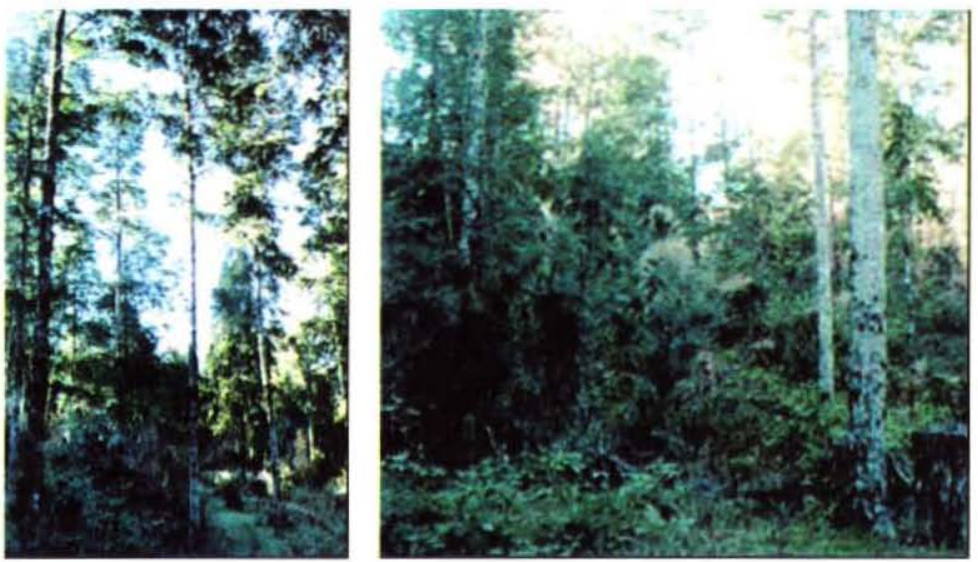

APS ROBLE FUY

Renoval roble, con participación menor de otras especies como tepa y coigüe.

2,1 ha

Predio Huilo Huilo

COFOMAP SA.

Comuna de Neltume

Región de los Rios

Salida del pueblo de Neltume hacia Fuy, a orilla del camino público. 


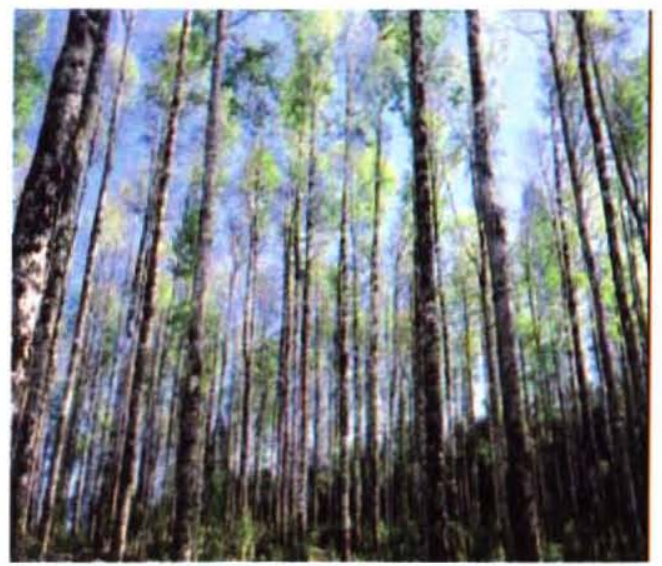

APS Roble PUMILLAHUE

Rodal roble 40 a 50 años edad

4 ha

Predio Pumillahue

CONAF

Comuna de Mariquina

Región de Los Ríos

İndice de calidad aumentó en un $25 \%$

Rodal aislado de polen externo, buenas

caracteristicas de forma.
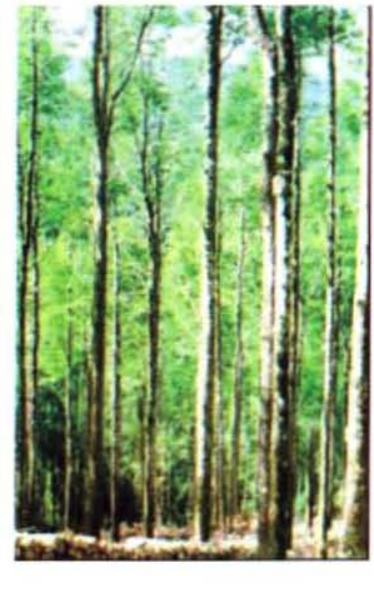

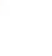




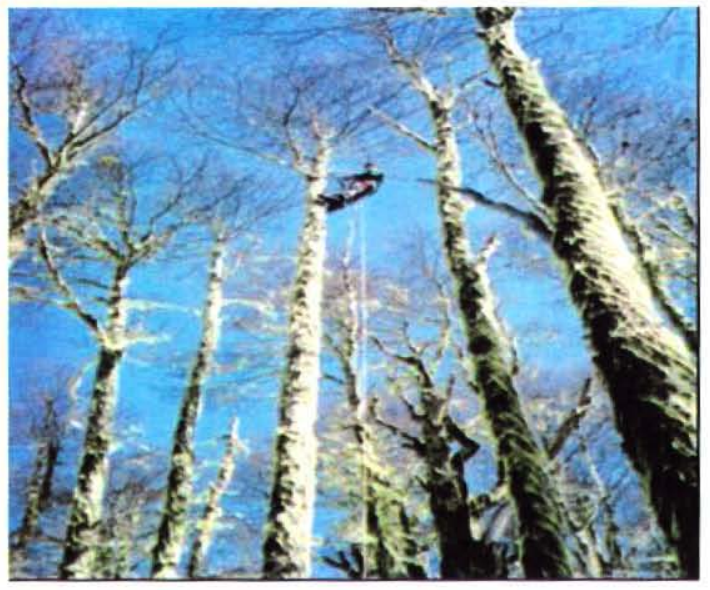

\section{APS Lenga CAIQUÉN GRANDE}

Rodal de lenga

6 ha

Predio El Cauquén Grande

Forestal Mininco SA.

Comuna de Coyhaique

Región de Aysén

Índice de calidad aumentó en un 22\%

Sector Vista Hermosa, $40 \mathrm{~km}$ al sur de Coyhaique.

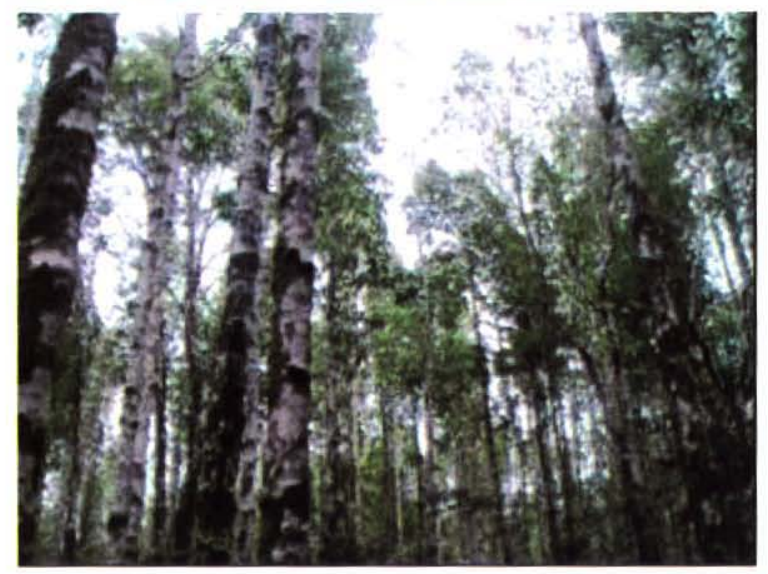

\section{APS Canelo RIO SUR}

Renoval casi puro canelo

2,5 ha

Predio Rio Sur José Soto Santana Comuna de Puerto Varas Región de Los lagos Sitio plano sobre suelos ñadis, a los pies del Volcán Calbuco.

Raleos sucesivos conducirian a incrementar Índice de calidad en un $37 \%$

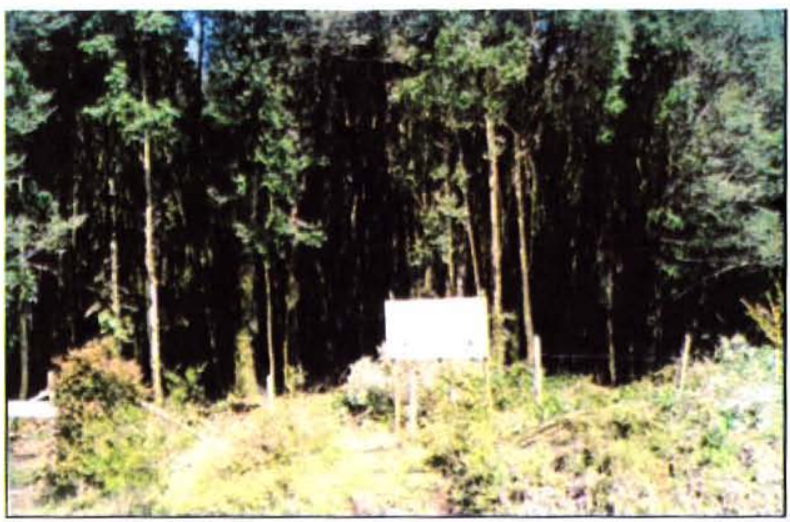

\section{APS Canelo AGUAS BUENAS}

Renoval de canelo 2 ha

Predio Aguas Buenas

Helga Montecinos

Comuna de Ancud

Isla de Chiloé Región de Los Lagos

Raleos sucesivos mejoran Índice de calidad en un $57 \%$

Suelos planos de ñadis. 

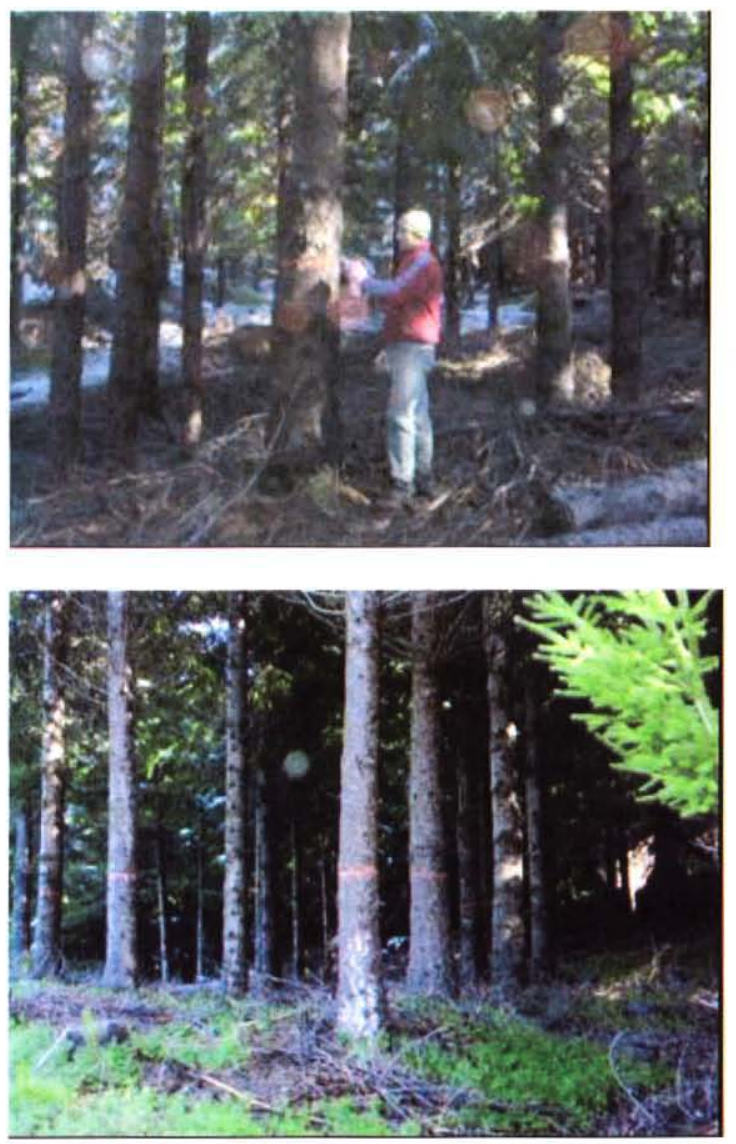

\section{APS Pino Oregón ARQUILHUE}

Plantación pino oregón año 1973 4,5 ha

Perdio Arquilhue

Agrícola y Forestal Taquihue Ltda. Comuna de Futrono

Región de Los Ríos

Índice de calidad aumenta en un 58\%

Rodal con raleos tardios (1995 y 2000) y suaves.

Suelos trumaos.

\section{APS Pino Oregón PILMAIQUÉN}

Plantación pino oregón 30 años

Predio Pilmaiquén

COFOMAP

Comuna de Neltume

Región de Los Ríos

Precordillera Andina

Área temporalmente reservada para reproducción de huemules, transformación en ASP pendiente.

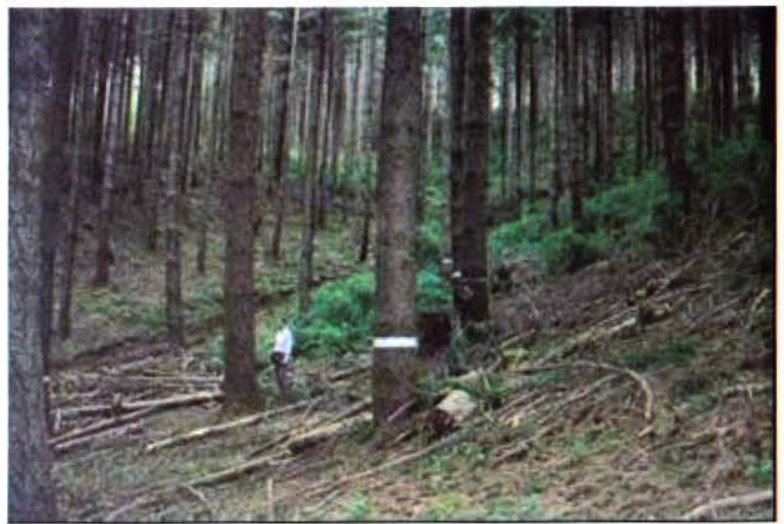

\section{APS Pino Oregón MALALCAHUELLO}

Plantación pino oregón año 12975

2,5 ha

Reserva Forestal Malalcahuello CONAF

Comuna de $i$ ?

Región de La Araucania Indice de calidad se incrementa en 65\%

Rodal con dos raleos previos. 

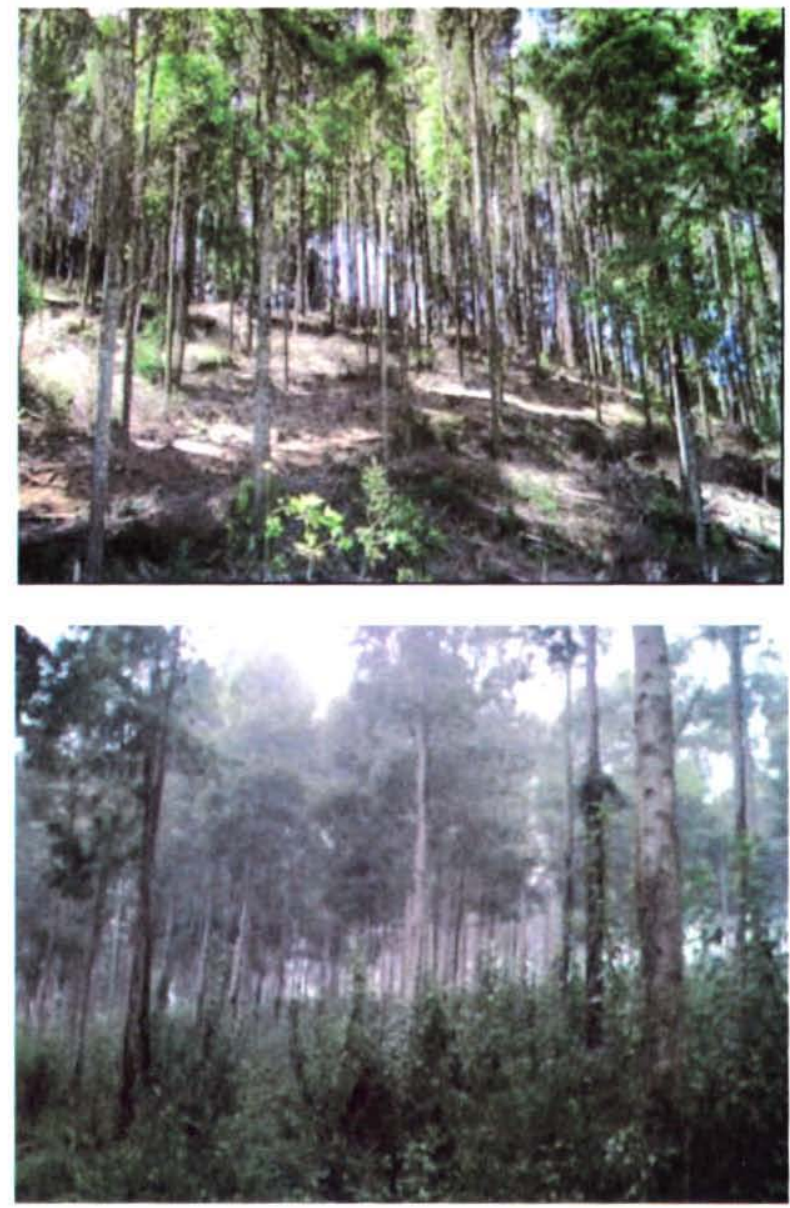

APS Acacia melanoxylon BUCHOCO

Rodal aromo australiano

1 ha

Sector Buchoco

Comuna de Contulmo

Región del Bio Bio

Ladera SW cercana a ribera

sur Lago Lanalhue

Rodal e regeneración natural

a partir de árboles cercanos de 18 a 20 años.

\section{APS Acacia melanoxylon QUI- LAS BAJAS}

Plantación aromo australiano 15 años edad

2 ha

Predio ¿? Quilas Bajas

Propietario Herman Toepser Klima y Otro

Comuna Freire

Región de la Araucanía

Ladera baja exposición NE

\section{REQUERIMIENTOS DE SEMILLA MEJORADA}

En el marco del proyecto Fondo SAG, Fuentes de Semilla Mejorada para las Especies Prioritarias en la Estrategia de Diversificación Forestal Nacional, se efectuó una evaluación de la demanda de semilla de las especies consideradas en el proyecto.

En primer lugar se estimó la producción de semilla en las Áreas Productoras de Semillas, especialmente las de Nothofagus, y posteriormente se efectuó una encuesta a viveros productores de las especies ubicados entre las Regiones del Maule y de Los lagos. 


\section{Disponibilidad de Semillas en APS}

La estimación de la producción de semillas de las distintas APS se efectuó se utilizando colectores de semillas de $1 \mathrm{~m}^{2}$, de malla puesta en forma de embudo (Figura $\mathrm{N}^{\circ} 3$ ), para capturar la semilla que cae en el periodo de fructificación y con esto tener una relación de la productividad de semilla por hectárea por cada APS. Se instaló 20 colectores por unidad productora de las especies de Nothofagus. Durante el primer año de evaluación, temporada 2004, la producción de semilla fue escasa a nula.

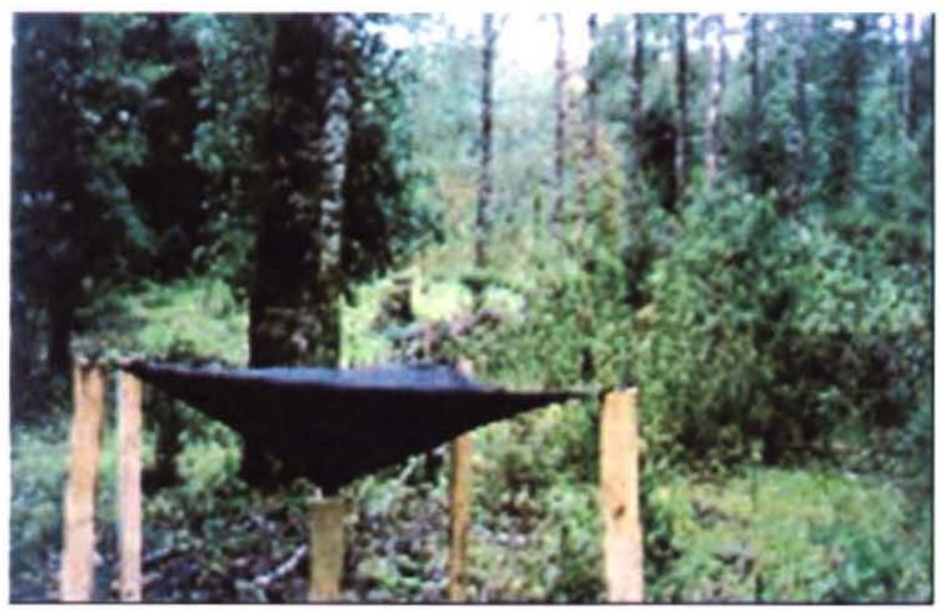

Figura $\mathrm{N}^{\circ} 20$

COLECTOR DE SEMILLA

Durante la temporada 2005-2006 se repitió la evaluación y, de acuerdo a las muestras obtenidas en los colectores, se estimó la producción de semillas por unidad de superficie, onteniéndose los resultados resumidos en el Cuadro $\mathrm{N}^{\circ} 5$.

\section{Cuadro $\mathrm{N}^{\circ} 5$}

ESTIMACIÓN DE LA PRODUCCIÓN DE SEMILLAS EN APS DE ROBLE, RAULI Y COIGÜE

\begin{tabular}{|l|r|r|}
\cline { 2 - 4 } \multicolumn{1}{c|}{} & \multicolumn{1}{|c|}{$\begin{array}{c}\text { Superficie Colectores } \\
\left(\mathbf{m}^{\mathbf{2}}\right)\end{array}$} & \multicolumn{2}{c|}{$\begin{array}{c}\text { Producción } \\
\mathbf{( K g / h a )}\end{array}$} \\
\hline ROBLE & 50 & 64,2 \\
\hline Rupanco & 1000 & 29,57 \\
\hline Arquilhue & 200 & 27,7 \\
\hline Pumillahue & 40 & 270,8 \\
\hline Puerto Fuy & & 1,35 \\
\hline RAULI & 20 & 0,58 \\
\hline Remeco & 20 & 4,3 \\
\hline Manzano & 40 & \\
\hline Malacahuello & 40 & \\
\hline
\end{tabular}




\begin{tabular}{|c|c|c|}
\hline & $\begin{array}{c}\text { Superficie Colectores } \\
\left(\mathrm{m}^{2}\right)\end{array}$ & $\begin{array}{c}\text { Producción } \\
\text { (Kg/ha) }\end{array}$ \\
\hline \multicolumn{3}{|l|}{ COIGUE } \\
\hline Pilmaiquén & 40 & 43,3 \\
\hline
\end{tabular}

En el caso de pino oregón la semilla fue cosechada directamente, escalando los árboles. Para ello se extrae los conos aún cerrados. No es conveniente dejar que la semilla precipite al suelo dado que es de fácil depredación. También se observó que en general en las APS de pino oregón un número minimo de árboles produce semilla. En este caso es más conveniente escalar aquellos árboles que presentan una producción adecuada ( $20 \%$ de la copa con presencia de conos).

La producción de semilla de las especies de Nothofagus es altamente variable de año en año, motivo por el cual es recomendable realizar una sobre cosecha en los años de buena producción y proceder a limpiar y a almacenar adecuadamente la semilla para temporadas posteriores.

Dentro de las APS de roble destaca la alta producción estimada para Puerto Fuy, la que se debe principalmente a que esta APS es una unidad en que el proceso de transformación se encuentra terminado y existe una mayor apertura del dosel y por ende un mayor volumen de copa y producción floral. Adicionalmente se puede agregar que la calidad del sitio es bastante apropiada, en términos de suelo (trumaos profundos) y situación de frio extremo. Esta última condición favorece la producción de semillas lo cual obedece a una estrategia de perpetuación de la especie.

También es posible concluir que la producción de semilla de rauli fue bastante baja. Se esperaba que fuera al menos similar a la de roble o coigüe. Con ello se concluye que los ciclos de producción para Nothofagus no son coincidentes para las especies del género.

En el caso de coigüe se detectó que es menos ciclico que el resto de los Nothofagus, pero la calidad de la semilla, en cuanto a viabilidad y germinacion, se ha revelado como altamente variable.

En el caso de lenga, no hubo producción en las temporadas evaluadas.

\section{Producción de Plantas en Viveros Forestales entre las Regiones del Maule y Los Lagos}

Con el fin de evaluar la demanda potencial de semilla de las especies forestales consideradas en el proyecto se realizó una encuesta alrededor de 100 viveros localizados, entre las Regiones del Maule y Los Lagos, y que tenian como caracteristica común la producción de una o más de las especies.

La mayor concentración de viveros se encontró en las Region de La Araucania (51). sin embargo la mayor producción de plantas de las especies estudiadas se encuentra en las Regiones de Los Rios y Los Lagos, con 5.126 .650 plantas de un total de 7.798 .364 plantas 
producidas por el total de los viveros encuestados.

En la Región del Maule se encuestó 15 viveros, entre los cuales se producen 7 de las especies de interès: aromo australiano, canelo, castaño, coigüe, pino oregón, rauli y roble. EI total de plantas producidas de estas especies en la Región es de 52.600 y corresponde sólo al $0,7 \%$ del total.

En la Región del Bio Bio se encuestó 25 viveros, los que producen en su conjunto las 10 especies consideradas en el proyecto. La Región produce 313.850 plantas de estas especies. lo que alcanza al el $4 \%$ del total nacional.

En las Regiones de Los Rios y Los Lagos, como se señaló anteriormente, se encuestó 51 viveros, pero se debió eliminar 9 , los cuales ya no producian las especies consideradas o bien ya no estaban en funcionamiento. Se produce 2.305.264 plantas de estas 10 especies, lo que corresponde al $30 \%$ del total.

En las cinco regiones encuestadas, el $59 \%$ de los viveros obtiene su semilla por cosecha directa (autoabastecimiento) o a través de proveedores informales. Sólo 7 de los viveros encuestados utiliza para su producción semilla mejorada proveniente de APS o Huerto Semillero. También se evidenció que el $10 \%$ de los viveros no utiliza semillas y produce a base de plantas extraidas de regeneración natural en rodales de las especies.

Respecto de problemas de disponibilidad de semilla, sólo el $25 \%$ de los viveros señaló tenerlos. Dentro de las causas, el $64 \%$ de los que señalan dificultades declara que la oferta de semilla es nula u ocasional. El resto de los viveros señaló que la dificultad se debe a que la semilla es muy cara.

En la pregunta que se relaciona con la existencia de una reglamentación legal para la semilla forestal que respalde el origen, calidad y sanidad, a nivel de las 4 regiones el $88 \%$ de los viveros, que respondieron la pregunta (95 viveros), señala que ello es muy importante porque de ese modo se asegura la obtención de mejores resultados en la producción de plantas con respecto a la inversión. A nivel regional esta respuesta se repite en el $100 \%$ de los viveros de la Región del Maule, el $91 \%$ de la Región del Bio Bio, el $89 \%$ de la Región de la Araucania y el $73 \%$ de las Regiones de Los Rios y Los Lagos. Los viveros que respondieron que no tiene mayor importancia este aspecto no fundamentan la respuesta o no confian en que una reglamentación en la materia efectivamente se cumpla.

A la consulta de si preferian utilizar semilla certificada por un organismo oficial, el $96 \%$ de los viveros encuestados responde afirmativamente. A nivel regional, estas respuestas son $100 \%, 95 \%, 100 \%$ y $87 \%$ en las regiones ordenadas de norte a sur.

Respecto del mismo tema, las respuestas positivas tampoco son fundamentadas claramente, pero en general señalan que podria ser un buen mecanismo para regular el precio de las semillas y avalar calidad y origen.

La especie que se produce en un mayor número de los viveros encuestados es pino 
oregón (50 viveros) y en el otro extremo está pino piñonero (4 viveros). Estas especies también representan los extremos en la producción de plantas. En las regiones involucradas se producen 2.673.380 de pino oregón y 4.300 de pino piñonero. En ambos casos, la mayor producción de plantas se produce en la Región de La Araucania y pino piñonero es producido sólo en las IX Regiones del Bio Bio y La Araucania.

Entre las especies de Nothofagus las más producidas son roble y coigüe, con 1.370 .830 y 1.343.110 planta, respectivamente, y estas especies concentran el $34,8 \%$ del total de producción de plantas del conjunto de especies consideradas en la encuesta. En menor escala se produce rauli, con el $13,6 \%$ de esta producción. En tanto lenga tiene una producción poco significativa, con el $2 \%$ de la producción total.

Para 9 de las 10 especies el mayor número de viveros se concentra en la Región de La Araucania. Solamente Eucalyptus regnans es producido mayoritariamente en la Región del Bio Bio. La producción de canelo, castaño, coigüe, lenga, rauli y roble está concentrada en la Región de La Araucania y, en particular la de especies de Nothofagus, sobre el $70 \%$ de la producción nacional, se realiza en la Región de La Araucania y concentrada en 17 viveros.

Al considerar las fuentes de abastecimiento de semillas, en la mayoria de las especies, sobre el $50 \%$ de los viveros utiliza semilla sin ningún grado de mejora ni certificación (autoabastecimiento o proveedores informales). Aparentemente sólo en coigüe, Eucalyptus regnans y pino oregón este porcentaje baja del $50 \%$ de los viveros, pero incluso en estas últimas sólo entre el 52 y $60 \%$ de los viveros utilizarian semilla de proveedores formales.

Al preguntar para qué especies se utiliza semilla mejorada (de APS o Huerto), se concluye que el número de viveros que lo hace para alguna es bastante escaso; 1 para aromo australiano, 2 para coigüe, 2 para rauli y 2 para roble. De acuerdo a los antecedentes que existen en INFOR, sólo habria un huerto clonal productivo de rauli, por lo que en el caso de las otras especies es probable que las semillas provengan de rodales o áreas que los viveristas estiman de buena calidad.

En términos generales, no se detectó problemas de importancia en abastecimiento de semillas, tal vez las mayores limitaciones estén en roble, rauli y coigüe, especies producidas en 35 a $39 \%$ de los viveros. En rauli y roble la oferta parece escasa y se debe principalmente a problemas de "añerismo". Para coigüe en tanto, los viveristas indican que las limitaciones están en baja oferta y alto costo de semilla.

Para las especies con menores limitaciones de disponibilidad los viveros se autoababstecen o lo hacen a través de proveedores informales. La excepción son Eucalyptus regnans y pino oregón, especies para las que la producción está fuertemente concentrada en un vivero de la Región del Bio Bio, para la primera, y en uno de la Región de La Araucania más dos de las Regiones de Los Rios y Los Lagos, para la segunda, y los viveristas aseguran su abastecimiento con fuentes formales. 


\section{COMENTARIOS Y CONCLUSIONES}

Las áreas productoras de semillas constituyen una alternativa válida para generar semilla genéticamente mejorada al inicio de un programa de mejoramiento genético y los rodales seleccionados, para su transformación en APS, efectivamente son de una calidad superior y cumplen con los estándares requeridos para conformar este tipo de fuentes semilleras.

La metodología empleada para la conversión de los rodales seleccionados en áreas productoras de semillas también resulta apropiada para este fin. De igual forma, la implementación de las intervenciones (raleos) se efectuó forma apropiada y las áreas productoras resultantes tienen un buen potencial para la producción de semilla genéticamente mejorada.

En términos de parámetros dasométricos, el estado actual de los rodales transformados enAPS es mejor que la situación proyectada al momento de su selección y evaluación originales. Ello obedece a la diferencia de tiempo que ha transcurrido entre la evaluación original y la actual, lo que ha significado un mayor crecimiento acumulado.

En términos de calidad, el escenario de las APS se ha presentado como intermedio entre las situaciones originales y proyectadas, siendo posible mejorar aún más el indice de calidad, como consecuencia de los raleos y depuraciones faltantes.

En sintesis las APS existentes constituyen una buena aproximación a la conformación de la red de semillas propuesta en el proyecto, restando aún cuantificar su producción por un periodo mayor e incluyendo aquellas APS que no han sido evaluadas aún en términos de su producción de semillas. Todo ello para determinar si estas son suficientes para satisfacer las demandas actuales y futuras de semilla mejorada que requerirá la diversificación forestal y la aplicación de la Ley de Bosque Nativo.

Es preciso destacar que las APS existentes corresponden a 7 de las 11 especies prioritarias para la diversificación forestal (roble, raulí, coigüe, lenga, canelo, aromo australiano y pino oregón). Para el caso de Pinus pinea, Castanea sativa y Eucalyptus regnans, el proyecto Fondo SAG propuso la conformación de Huertos Semilleros Clonales a partir de árboles plus seleccionados durante la ejecución del mismo. Actualmente, ya se ha instalado los huertos para castaño y Eucalyptus regnans. En el caso de Pinus pinea, no ha sido posible obtener los rametos (clones injertados) suficientes para su implementación. 